\title{
Eventual re-vitrification or storage in liquid nitrogen vapor does not jeopardize the practical handling and transport of vitrified pig embryos
}

Alicia Nohalez, Cristina A. Martinez, Inmaculada Parrilla, Carolina Maside, J ordi Roca, Maria A. Gil, Heriberto Rodriguez-Martinez, Emilio A. Martinez and Cristina Cuello

The self-archived postprint version of this journal article is available at Linköping University Institutional Repository (DiVA):

http:/ / urn.kb.se/ resolve?urn=urn:nbn:se:liu:diva-147898

N.B.: When citing this work, cite the original publication.

Nohalez, A., Martinez, C. A., Parrilla, I., Maside, C., Roca, J., Gil, M. A., Rodriguez-Martinez, H., Martinez, E. A., Cuello, C., (2018), Eventual re-vitrification or storage in liquid nitrogen vapor does not jeopardize the practical handling and transport of vitrified pig embryos, Theriogenology, 113, 229-236. https:// doi.org/ 10.1016/j.theriogenology.2018.03.001

Original publication available at:

https:// doi.org/ 10.1016/j.theriogenology.2018.03.001

Copyright: Elsevier

http:// www.elsevier.com/

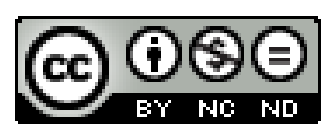


3 Eventual re-vitrification or storage in liquid nitrogen vapor does not jeopardize

6 Alicia Nohalez ${ }^{\mathrm{a}, \mathrm{b}}$, Cristina A. Martinez ${ }^{\mathrm{a}, \mathrm{b}}$, Inmaculada Parrilla ${ }^{\mathrm{a}, \mathrm{b}}$, Jordi Roca ${ }^{\mathrm{a}, \mathrm{b}}$, María A.

$7 \quad$ Gil $^{\mathrm{a}, \mathrm{b}}$, Heriberto Rodriguez-Martinez ${ }^{\mathrm{c}}$, Emilio A. Martinez ${ }^{\mathrm{a}, \mathrm{b}^{*}}$, Cristina Cuello ${ }^{\mathrm{a}, \mathrm{b}}$

8

$9{ }^{a}$ Faculty of Veterinary Medicine, International Excellence Campus for Higher 10 Education and Research “Campus Mare Nostrum”, University of Murcia, Murcia, Spain

11 bInstitute for Biomedical Research of Murcia (IMIB-Arrixaca), Murcia, Spain

12 'Department of Clinical \& Experimental Medicine (IKE), Linköping University, 13 Linköping, Sweden

\section{*Corresponding author}

Emilio A. Martinez

17 Facultad de Veterinaria. Campus de Espinardo, 30100, Murcia, Spain

E-mail: emilio@um.es

Tel.: +34 868884734

Fax: +34 868887069 


\section{Abstract}

2 This study aimed (1) to evaluate the in vitro post-warming survival of porcine embryos

3 after re-vitrification and (2) to assess the efficacy of transport of embryos in dry shipper

4 (DS) in maintaining the viability and quality of vitrified embryos for a 3-day period.

5 Embryos at the compacted or cavitating morula (CCM) and unhatched blastocyst (UBL)

6 stages were surgically obtained from weaned, crossbred sows. In the first experiment,

7 more than $85 \%$ of the embryos survived an initial vitrification and warming and

8 achieved comparable survival rates to those of their fresh counterparts. In contrast, those

9 embryos subjected to a second vitrification and warming had clearly lower survival

10 rates $(60 \%$ and $64 \%$ for re-vitrified embryos from the CCM and UBL groups,

11 respectively) compared to the survival rates of the initial vitrification and fresh control groups $(\mathrm{P}<0.01)$. Hatching rates were similar in re-vitrified blastocysts derived from vitrified CCMs and fresh control groups (50.8\% and 55.3\%, respectively). However, differences $(\mathrm{P}<0.01)$ in hatching rates were recorded in re-vitrified blastocysts derived from vitrified UBLs and fresh control blastocysts (14.7\% and 90.0\%, respectively). In the second experiment, vitrified embryos were stored in a liquid nitrogen tank for one month. Then, the straws containing the embryos were transferred to a DS (DS group) or to another liquid nitrogen tank (control group) for an additional three days. Embryos from the DS and control groups had similar survival and hatching rates, regardless of the embryonic stage considered. The DS storage of CCMs and UBLs did not affect their development after culturing, including total cell numbers, compared to the control, although their apoptotic index was slightly higher $(\mathrm{P}<0.05)$, regardless of the developmental stage. In conclusion, although re-vitrification negatively affects embryo survival, this study demonstrated that $>60 \%$ of vitrified embryos could be successfully 
1 re-vitrified and re-warmed. The present study also showed the effectiveness of the DS

2 for the storage of vitrified porcine CCMs and UBLs for at least three 3 days.

4 Keywords: Morulae; Blastocyst; Double vitrification; Dry shipper; Porcine.

\section{1. Introduction}

7 The competitiveness of the swine industry is under pressure globally. In this regard,

8 ample use of reproductive technologies is of vital importance, particularly considering

9 embryo transfer (ET). ET technology has been a demand of the pig industry for more

10 than 60 years due to its numerous applications, particularly for the safe exchange of

11 high-value genetic material with reduced transportation costs. Moreover, this technique

12 substantially diminishes animal welfare problems. Despite these advantages, the commercial use of ET in pigs is still very limited when compared with other species [1]. The need to use surgical procedures for embryo collection and the difficulties encountered in embryo cryopreservation in pigs have led to a loss of interest in this technology for decades. However, in recent years, the situation has changed thanks to the technological development of better vitrification procedures [2] and of a safe, nonsurgical ET [1].

In ET programs, the embryos must be stored from the time of their collection until they can be transferred to the recipients. Today, the only effective method for long-term preservation of porcine embryos is vitrification, which avoids the formation of intracellular ice crystals [3]. The improvement of vitrification procedures, specifically by using the open pulled straw (OPS) method [4], has provided high in vitro postwarming embryo survival rates and hopefully higher pregnancy and farrowing rates after surgical ET of in vivo-derived porcine embryos vitrified without any previous 
1 treatment [5]. Moreover, recent studies clearly showed similar survival rates after $24 \mathrm{~h}$

2 in culture between vitrified and fresh embryos [6].

3 Although major progress has occurred in porcine embryo vitrification, many factors still

4 remain unsolved. Of particular interest among these factors are embryo re-vitrification

5 and air embryo shipment in the vapor phase of liquid nitrogen; these practical questions

6 are of utmost importance since they are probably the weakest links of the ET chain.

7 In some circumstances during ET, such as when the number of warmed embryos

8 exceeds the number of embryos necessary to be transferred to the recipients or when

9 some recipients cannot receive embryos due to health problems or difficulties during the

10 insertion of the non-surgical ET catheter, a number of unexpected supernumerary

11 embryos could have been warmed. Although these extra warmed embryos could be used to increase the number of transferred embryos per recipient, the ideal would be that they could be re-vitrified and stored again for future ET. In this sense, the possibility of revitrification should provide more flexibility for transfer to the respective recipients. In addition, re-vitrification could be used as an embryo stress resistance and quality test as suggested by Isachenko et al. [7]. Recent studies have used re-vitrification procedures with considerable success for human [8,9], ovine [10] and murine [7] embryos. However, the effects of re-vitrification on post-warming embryo survival rates in pigs have not yet been elucidated.

The shipment of embryos in liquid nitrogen appears optimal for long-distance transportation of vitrified pig embryos [11]. However, a major practical concern is the decision by the International Air Transport Association (IATA) to forbid the transport of tanks with liquid nitrogen. Since then, the only option for the air transport of cryopreserved embryos is to use special containers, the so-called dry shippers (DSs). 
1 hydrophobic membrane. The absorbent material repels humidity but absorbs liquid

2 nitrogen, allowing maintenance of a vapor phase of liquid nitrogen at $-150{ }^{\circ} \mathrm{C}$ [12].

3 Although this temperature appears to be adequate for the safe shipment of biological

4 specimens that are conventionally frozen, it could be critical for vitrified porcine

5 embryos, which are particularly sensitive to temperature variations [11]. Vitrified

6 porcine embryos are traditionally stored at $-196{ }^{\circ} \mathrm{C}$ in very small volumes of medium

7 and, therefore, an oscillation in the storage temperature can induce medium

8 devitrification, ice crystal growth and the death of the embryos. The DS Dewars have

9 been used for cryopreserved human semen [13,14] and oocytes [15] and for vitrified

10 mouse embryos [16]. However, to the best of our knowledge, there are no published

11 studies on the effectiveness of dry shippers for the transport of vitrified porcine

12 embryos.

13 The aims of this study were to (1) evaluate the in vitro survival of in vivo-derived 14 porcine embryos at the compacted or cavitating morula (CCM) and unhatched 15 blastocyst (UBL) stages after re-vitrification and (2) to assess the effectiveness of the 16 DSs in maintaining the viability and quality of vitrified in vivo-derived porcine CCMs 17 and UBLs for a 3-day storage period.

2. Methods

\subsection{Chemicals}

22 All chemicals used in these experiments were purchased from Sigma-Aldrich Quimica SA (Madrid, Spain) unless otherwise noted.

\subsection{Animals}


1 The embryos were obtained under field conditions at two commercial farms located in

2 southeastern Spain (Agropor SA, and Porcisan SA, Murcia, Spain). Crossbred Landrace

3 x Large-White sows (parity 2 to 6) with normal weight and sanitary status were used in

4 the experiments. Sows were individually allocated into crates in a mechanically

5 ventilated confinement facility. Animals were fed a commercial ration according to their

6 nutritional requirements. All sows had water available ad libitum. Boars were housed in

7 individual pens in a commercial artificial insemination center (AIM Iberica, Murcia,

8 Spain).

9 All experimental procedures were performed in accordance with the 2010/63/EU EEC

10 directive for animal experimentation and were reviewed and approved in advance by the

11 Ethical Committee for Experimentation with Animals of the University of Murcia,

12 Spain (research code: 183/2015).

\subsection{Detection of estrus and artificial insemination}

The embryo donors were examined (beginning the day after weaning) for estrus twice a day by exposing sows to a mature boar and applying back pressure. Sows in estrus were post-cervically inseminated at 4 and $24 \mathrm{~h}$ after the onset of estrus. Insemination doses $\left(1.5 \times 10^{9}\right.$ spermatozoa in $\left.45 \mathrm{~mL}\right)$ were prepared in the AI center from ejaculates diluted in BTS extender (Beltsville Thawing solution) [17]. Sperm doses were conserved for a maximum of $24 \mathrm{~h}$ at $18^{\circ} \mathrm{C}$.

\subsection{Embryo recovery}

Surgeries were performed by mid-ventral laparotomy on day 6 of the estrous cycle, considering day 0 to be the onset of estrus. Donors were previously sedated with azaperone (Stresnil® ${ }^{\circledR}$, Landegger Strasse, Austria; 2 mg/kg body weight, i.m.). General 
1 anesthesia was induced with sodium thiopental (B. Braun VetCare SA, Barcelona,

2 Spain; $7 \mathrm{mg} / \mathrm{kg}$ body weight, i.v.) and maintained with isoflurane (IsoFlo ${ }^{\circledR}$, Madrid,

3 Spain). The reproductive tract was exposed and the corpora lutea in each ovary were

4 counted. Embryos were collected as described previously [18] by flushing the tip of

5 each uterine horn with $30 \mathrm{~mL}$ of Tyrode's lactate (TL)-HEPES-polyvinyl alcohol

6 (PVA) $[18,19]$

7

\section{$8 \quad$ 2.5. Embryo assessment}

9 The recovered embryos were evaluated under a stereomicroscope for developmental 10 stage and quality. One-cell eggs or poorly developed embryos were considered unfertilized oocytes or degenerated embryos, respectively. Embryos with an appropriate morphology according to the criteria determined by the International Embryo Transfer Society [20] were classified as viable. Vitrification was only performed on viable CCMs (embryos with blastomeres totally compacted and an undistinguishable cell boundary or with initial cavitation and UBLs (embryos with a differentiated blastocoele, inner cell mass and trophpoblast).

\subsection{Vitrification and warming}

The embryos were washed three times with TL-HEPES-PVA medium before vitrification and maintained at $39{ }^{\circ} \mathrm{C}$. Vitrification was performed within $4 \mathrm{~h}$ after embryo recovery. Groups of three to six embryos were vitrified by the method described by Cuello et al. [21]. Briefly, the embryos were washed twice in TL-HEPESPVA medium and sequentially equilibrated in the first vitrification medium (TLHEPES-PVA+7.5\% dimethyl sulfoxide+7.5\% ethylene glycol) for $3 \mathrm{~min}$ and in the second vitrification medium (TL-HEPES-PVA+16\% dimethyl sulfoxide+16\% ethylene 
1 glycol+0.4 M sucrose) for $1 \mathrm{~min}$. Finally, the embryos were placed in a 1.0 to $1.5 \mu \mathrm{L}$

2 drop and loaded into the narrow end of a superfine open pulled straw (SOPS; Minitüb,

3 Tiefenbach, Germany) by capillary action. Immediately, the straw containing the

4 embryos was plunged horizontally into liquid nitrogen.

5 After storage, embryos were warmed in a four-well multi-dish plate (Nunc A/C, 6 Roskilde, Denmark). Briefly, the straw containing the embryos were removed from de 7 liquid nitrogen and immediately (less than one second) vertically submerged in the well 8 containing $1 \mathrm{~mL}$ of TL-HEPES-PVA at $39{ }^{\circ} \mathrm{C}$ supplemented with $0.13 \mathrm{M}$ sucrose, for 95 min. Finally, embryos were placed in another well containing $800 \mu \mathrm{L}$ of TL-HEPES10 PVA.

\subsection{In vitro embryo culture and evaluation of in vitro development post-warming}

Post-warmed embryos were cultured in a four-well multi-dish plate containing $500 \mu \mathrm{L}$ of embryo culture medium (NCSU-23) [22] supplemented with $0.4 \%$ bovine serum albumin (BSA) and $10 \%$ fetal calf serum at $39{ }^{\circ} \mathrm{C}$ in humidified air with $5 \% \mathrm{CO}_{2}$. The embryos were evaluated morphologically with a stereomicroscope to determine their viability and developmental stage. Compacted or cavitating morulae that had progressed to the blastocyst stage during in vitro culture and UBLs that reformed their blastocoelic cavities post-warming with an excellent or good appearance were considered viable. Survival rate was determined as the ratio of viable embryos to the total number of cultured embryos. Hatching rate was defined as the ratio of hatching and hatched embryos at the end of the culture to the total number of cultured embryos.

\subsection{Assessment of cellular apoptosis and total cell number in blastocysts}


1 Apoptotic cells were stained by terminal deoxynucleotidyl transferase and fluorescein

2 dUDP nick-end labelling (TUNEL) as described in Brison and Schultz [23], and Byrne

3 et al. [24], using an $A P O-B r d U^{T M}$ TUNEL Assay Kit (A23210; Invitrogen, Oregon,

4 USA). Briefly, viable blastocysts were fixed in 4\% paraformaldehyde and washed in

5 phosphate buffer solution (PBS)-BSA (3 mg/mL). Then, embryos were permeabilized

6 overnight at $4{ }^{\circ} \mathrm{C}$ using $1.5 \%$ Triton $\mathrm{X}-100$ and $0.15 \%$ Tween 20 in PBS. Afterwards,

7 blastocysts were washed in PBS-BSA for $10 \mathrm{~min}$. Fixed and permeabilized embryos

8 from the same donors were used as positive control. After permeabilization, positive

9 control embryos were incubated in DNase I (50 U/mL) in PBS-BSA for $20 \mathrm{~min}$ at $39^{\circ} \mathrm{C}$

10 in a petri-dish covered with mineral oil. Then, both control and experimental embryos

11 were placed in PBS-BSA with $0.5 \%$ Tween 20 for 10 min and incubated in TUNEL

12 reaction droplets $(10 \mu \mathrm{L})$ for $1 \mathrm{~h}$ at $38.5{ }^{\circ} \mathrm{C}$ in the dark. Finally, all the embryos were washed in PBS-BSA three times for 5 min each time at room temperature and mounted on a slide in droplets with Hoechst (33342)-Vectashield $(10 \mu \mathrm{g} / \mathrm{mL})$. Samples were examined under a fluorescence microscope (Eclipse E800, Nikon, Japan) using excitation filters of $465-495 \mathrm{~nm}$ and $330-380 \mathrm{~nm}$ for green and blue fluorescence, respectively. The total number of nuclei that displayed blue fluorescence was counted. Cells were considered TUNEL positive when their nuclei exhibited green fluorescence. Blastocysts were considered undamaged when they did not show TUNEL positive nuclei or areas. The percentage of apoptotic cells was calculated as TUNEL positive nuclei out of the total number of nuclei stained with Hoechst (apoptotic index).

\subsection{Dry shipper features and management}

The DS used was an ST Repro 6.4 model (Reproductive Technologies, Navasota, Texas) that holds $6.4 \mathrm{~L}$ of liquid nitrogen total capacity, $4.2 \mathrm{~L}$ of liquid nitrogen 
1 absorbed, and has the capacity to hold 1,600 $0.25 \mathrm{~mL}$-straws. Twenty-four hours before

2 the beginning of the experiment, the Dewar was gradually pre-chilled, adding small

3 amounts of liquid nitrogen into the canister chamber to avoid excessive bubbling, until

4 it was apparently full. To reach full absorption (a weight of $10.6 \mathrm{~kg}$ ), the canister

5 chamber was refilled 2 h later. The Dewar was maintained overnight at $4{ }^{\circ} \mathrm{C}$. Finally,

6 the canister chamber was refilled once again. Two hours later, the remaining liquid

7 nitrogen was removed, and the straws containing the embryos were transferred from the

8 liquid nitrogen tank to the DS. The temperature was checked within the canister to

9 confirm that it was approximately $-150^{\circ} \mathrm{C}$.

\subsection{Statistical analysis}

12 Statistical analyses were performed using IBM SPSS 19 Statistics (SPSS, Chicago, IL, USA). The data expressed in percentages were analyzed by Fisher's exact test. Continuous variables were evaluated using the Kolmogorov-Smirnov test to assess the assumption of normality, and groups were compared using unpaired Student's t-tests corrected for inequality of variances (Levene's test). The threshold for significance was set at $\mathrm{P}<0.05$. Results are expressed as percentages and least square means \pm standard deviation (SD).

\section{Results}

A total of 26 donor sows were used in this study. The mean ovulation rate in these sows was $21.1 \pm 3.9$. The ratio of collected embryos to the number of corpora lutea was $93.9 \%$. The total number of oocytes and embryos collected was 501, of which $3.2 \%$ were unfertilized oocytes and degenerated embryos, $45.7 \%$ were CCMs, $45.7 \%$ were UBLs and $5.4 \%$ were hatched blastocysts. 
2 This experiment was performed to evaluate the effects of the re-vitrification of warmed

3 embryos on the in vitro embryo survival. For that, collected embryos $(\mathrm{N}=255)$ were

\subsection{Experiment 1} assigned to one of two groups: a group that underwent a single vitrification and warming (vitrification control group) and a fresh embryo control group. Each group was thereafter divided into two sub-groups according to their embryonic developmental stage (CCM or UBL). Embryos from the fresh control group were not vitrified, and embryo viability was evaluated at $24 \mathrm{~h}$ of in vitro culturing. Viability and hatching rates of embryos from the single vitrification group were evaluated at $24 \mathrm{~h}$ post-warming. Subsequently, unhatched viable blastocysts were re-vitrified and re-warmed $(\mathrm{N}=136)$ and the viability of those embryos was assessed at $24 \mathrm{~h}$ of in vitro culturing. Finally, we evaluated hatching rates of re-vitrified and fresh control embryos at $48 \mathrm{~h}$ of in vitro culturing (see Fig. 1, for schematic representation of the experimental design).

In vitro survival rates of vitrified and re-vitrified embryos at $24 \mathrm{~h}$ post-warming are presented in Table 1. A high proportion $(>85 \%)$ of embryos survived the first vitrification and warming and achieved comparable survival rates to those of their fresh counterparts. At 24 h post-warming the $6.4 \%$ and $20.8 \%$ of blastocysts derived from vitrified CCMs and UBLs, respectively, were hatched (Fig. 2). These embryos were considered as viable but were not used for subsequent vitrification. A subsequent second vitrification and warming was harmful to the embryos since their survival rates were significantly decreased $(\mathrm{P}<0.01)$, regardless of embryonic stage, when compared with those of the control groups. At $48 \mathrm{~h}$ of culturing, hatching rates were similar in revitrified blastocysts derived from vitrified CCMs and fresh control groups (50.8\% and 55.3\%, respectively). Significant differences $(\mathrm{P}<0.01)$ in hatching rates were recorded 
1 in re-vitrified blastocysts derived from vitrified UBLs and fresh control blastocysts

$2 \quad$ (14.7\% and 90.0\%, respectively; Fig. 3).

3

\section{$4 \quad$ 3.2. Experiment 2}

5 This experiment evaluated the efficacy of transport of embryos in a DS dewar in 6 maintaining the viability of vitrified embryos for a 3-day storage period. After

7 vitrification, SOPS straws containing the embryos were stored in a liquid nitrogen tank

8 for one month. Then, the straws were transferred from the liquid nitrogen tank to the DS

9 (DS group; $\mathrm{N}=102$ ) or to another liquid nitrogen tank (control group; $\mathrm{N}=101$ ) for an

10 additional three days. In both groups, the embryos were divided according to their

11 developmental stage (CCM or UBL). After warming, embryos from the DS and the control groups were cultured for 24 h to assess embryo survival and hatching rates. Finally, an aliquot of viable embryos from each group was subjected to a TUNEL assay to evaluate nuclei with DNA degradation and the apoptotic index. (see Fig. 4, for schematic representation of the experimental design).

Figure 5 shows the survival rates of CM-CAVs and UBLs stored for three days in the DS. Embryos from the DS group presented similar survival rates to those of the liquid nitrogen control group (range: 88-100\%), regardless of the embryonic stage tested. The storage of CCMs and UBLs in DS did not affect embryo development towards the end of culture compared to the control group (Fig. 6A). The pattern of the frequency distribution of embryonic development was comparable between the groups (Fig. 6B). Although there were no differences in the total cell number of the resulting blastocysts between the DS and liquid nitrogen groups, the apoptotic index was affected, being slightly higher $(\mathrm{P}<0.05)$ in those embryos stored in the DS, regardless of the developmental stage considered (Fig. 7). 


\section{4. Discussion}

2 This is probably the first study detailing the embryonic survival and development of 3 porcine CCMs and UBLs subjected to double vitrification. The present study also

4 shows, for the first time, the effectiveness of DS Dewars for the storage of vitrified 5 porcine CCMs and UBLs for a three-day period, which is of major practical importance 6 for the commercial air transportation of embryos.

7 Previous investigations have demonstrated that the success of vitrification depends on

8 the embryonic developmental stage and that embryos at the morula and blastocyst

9 stages are more resistant to cooling than earlier stages and are therefore more suitable 10 for vitrification $[2,11,25]$. In the present study, the post-warming survival rates of 11 CCMs and UBLs were comparable to those achieved in previous studies [26-28]. Taken 12 together, the results from experiments 1 and 2 indicated that approximately $90 \%$ and 95\% of CCMs and UBLs, respectively, survived vitrification. These results are consistent with the general assumption that blastocysts usually have higher survival rates than morulae $[6,28]$. However, in the second experiment, we did not observe differences in survival rates between the two developmental stages. This observation is not surprising because similar results had previously been reported [21]. The use of morulae in different stages of development (uncompacted, partially compacted, fully compacted or with initial cavitation) might be responsible for that apparent discrepancy. In sum, our results confirm those of earlier studies [27], namely, that the SOPS 21 vitrification procedure is able to maintain the post-warming viability of vitrified morulae and blastocysts without any previous treatment. Moreover, an adequate in vivo developmental capacity of these embryos following embryo transfer has also been reported $[29,30]$. 
1 Re-vitrification has been successfully accomplished in murine [7,31], and human

2 embryos [9]. These studies demonstrated no differences in the post-warming survival

3 rates of mouse, rat and human embryos between the first and second vitrification or in

4 human pregnancy rates between re-vitrified and control embryos. To the best of our

5 knowledge, this is the first study where re-vitrification of porcine embryos has been

6 attempted. Our results indicate that although high survival rates were achieved after the

7 first vitrification process, a subsequent second vitrification was detrimental to embryo

8 viability since the post-warming survival rates in embryos from the re-vitrified group

9 were lower than those of the single vitrification and fresh control groups. These results

10 contrast with the abovementioned studies in murine and human embryos. The reasons

11 for this discrepancy are not clear but are probably linked to species-specific differences

12 in the sensitivity of embryos to low temperatures. It is known that porcine embryos, which contain high levels of intracellular lipids, are particularly sensitive to thermal effects [32,33].

Surprisingly, re-vitrified blastocysts derived from vitrified CCMs had higher postwarming hatching rates at the end of the culture period than re-vitrified blastocysts derived from vitrified UBLs. The effects of a lengthy period (aging) during the first and second post-warming cultures of vitrified and re-vitrified blastocysts could be one of the main explanations of these results since, as suggested by Hardy et al. [34], culture time markedly increases embryonic cell death.

Despite the detrimental effects of re-vitrification, approximately $60 \%$ of the embryos survived the second vitrification. Although the data suggest the possibility for cryopreservation of unexpected, extra warmed embryos for future re-warming and transfer, further studies are required to evaluate the real potential of transferring revitrified porcine embryos. 
1 In the second experiment, we did not find differences in embryo viability at $24 \mathrm{~h}$ post-

2 warming between vitrified embryos stored for three days in a DS or in a liquid nitrogen

3 tank. These results are in accordance with those of AbdelHafez et al. [16], who did not

4 find a detrimental effect of storage when mouse embryos (8-cell and blastocyst stages)

5 were stored in a DS for 4 days. Moreover, the results demonstrated that storage in a DS

6 neither affected the survival rate nor produced a delay in the subsequent embryo

7 development. Hatching rates were similar in the DS and control groups, supporting that

8 assumption. Although the evaluation of embryo hatching rates after in vitro culture of

9 the vitrified-warmed embryos has been proposed as a valid assessment of the efficiency

10 of vitrification and as a suitable parameter for the estimation of the quality of those

11 blastocysts in absence of ET [35], an additional assessment of cell death was conducted in our study. The TUNEL assay of broken DNA has been used in numerous cell types to detect the final stages of cell apoptosis [36]. Previous studies have demonstrated that the apoptotic index of in vivo-derived fresh porcine blastocysts is approximately $1 \%$ $[35,37]$. This suggests that apoptosis occurs in a small number of blastomeres as a natural process to remove unnecessary or damaged individual cells. In the present study, the apoptotic index in the control embryos (embryos stored in the liquid nitrogen tank) was comparable to that reported in the literature for fresh embryos $[34,36]$. However, the apoptotic index in embryos stored in the DS was 1.5 times greater. These results contrast with those reported by AbdelHafez et al. [16], in mouse embryos, who did not find an increase in the apoptotic index of embryos stored in a DS for 4 days. Differences among species in the sensitivity of embryos to temperature fluctuations [32,33] could explain the discrepancy. Although the apoptotic index values were below 3\% and likely have little biological significance, further studies are necessary to verify whether these values have some consequences for in vivo embryo development. 


\section{4.1. Conclusions}

3 In conclusion, although re-vitrification was detrimental to a significant proportion of

4 embryos, our results indicate that more than $60 \%$ of porcine blastocysts derived from

5 vitrified and warmed CCMs and UBLs survive re-vitrification and re-warming.

6 Therefore, embryos that were either fortuitously warmed or those warmed embryos that

7 were not transferred into recipients due to special circumstances may be re-vitrified and

8 used in future ETs. This study also shows the efficacy of transport of embryos in the DS

9 for short storage (three days) of vitrified CCMs and UBLs. The use of DS Dewar should

10 allow the safe worldwide air transport of porcine embryos. However, further studies are

11 needed to evaluate the in vivo results after ET.

\section{Acknowledgments}

The authors are grateful to Moises Gonzalvez for his assistance throughout this work.

We thank the Ministry of Economy and Competitiveness (Madrid, Spain) for its grantbased support of A Nohalez and CA Martinez (BES-2013-064069 and BES-2013064087, respectively).

\section{Funding}

This study was supported by the Ministry of Economy and Competitiveness (Madrid, Spain)/the European Regional Development Fund (grant number AGL2015-69735-R), and the Seneca Foundation, Murcia, Spain (grant number 19892/GERM/15). 


\section{Role of the funding source}

2 Funding sources did not have any involvement in the study design, in the collection,

3 analysis and interpretation of data, in the writing of the report, and in the decision to

4 submit the article for publication.

5

6 Author contributions

7 A Nohalez, H Rodriguez-Martinez, EA Martinez and C Cuello, conceived, designed and 8 directed the study. A Nohalez, CA Martinez, I Parrilla, MA Gil, EA Martinez and C

9 Cuello performed the experiments. A Nohalez, EA Martinez and C Cuello analyzed and 10 interpreted the data. A Nohalez, EA Martinez and C Cuello wrote the manuscript. J 11 Roca and H Rodriguez-Martinez critically revised the manuscript. All authors approved 12 the manuscript for publication.

\section{Declaration of interest}

None of the authors have any conflicts of interest to declare.

\section{References}

[1] Martinez EA, Cuello C, Parrilla I, Martinez CA, Nohalez A, Vazquez JL, et al. Recent advances toward the practical application of embryo transfer in pigs. Theriogenology 2016;85:152-61.

[2] Sanchez-Osorio J, Cuello C, Gil MA, Almiñana C, Parrilla I, Caballero I, et al. Factors affecting the success rate of porcine embryo vitrification by the Open Pulled Straw method. Anim Reprod Sci 2008;108:334-44.

[3] Vajta G, Kuwayama M. Improving cryopreservation systems. Theriogenology 2006;65:236-44. 
1 [4] Vajta G, Holm P, Greve T, Callesen H. Vitrification of porcine embryos using the Open Pulled Straw (OPS) method. Acta Vet Scand 1997;38:349-52.

[5] Martinez EA, Cuello C, Parrilla I, Rodriguez-Martinez H, Roca J, Jose L, et al. Current progress in non-surgical embryo transfer with freshand vitrified/warmed pig embryos. In: Rodriguez-Martinez, H, Soede, N.M., Flowers, W.N. Leicestershire, UK: Control of Pig Reproduction IX, 2013;101-12.

[6] Cuello C, Gil MA, Parrilla I, Tornel J, Vázquez JM, Roca J et al. Vitrification of porcine embryos at various developmental stages using different ultra-rapid cooling procedures. Theriogenology 2004;62:353-61.

[7] Isachenko V, Folch J, Isachenko E, Nawroth F, Krivokharchenko A, Vajta G, et al. Double vitrification of rat embryos at different developmental stages using an identical protocol. Theriogenology 2003;60:445-52.

[8] Chang CC, Shapiro DB, Bernal DP, Wright G, Kort HI, Nagy ZP. Two successful pregnancies obtained following oocyte vitrification and embryo revitrification. Reprod Biomed Online 2008;16:346-49.

[9] Kumasako Y, Otsu E, Utsunomiya T, Araki, Y. The efficacy of the transfer of twice frozen-thawed embryos with the vitrification method. Fertil Steril 2009;91:383-86.

[10] Leoni G, Bogliolo L, Pintus P, Ledda S, Naitana S. Sheep embryos derived from FSH/eCG treatment have a lower in vitro viability after vitrification than those derived from FSH treatment. Reprod Nutr Dev 2001;41:239-46.

[11] Dobrinsky, JR. Advancements in cryopreservation of domestic animal embryos. Theriogenology 2002;57:285-302. 
1 [12] Bielanski, A. Non-transmission of bacterial and viral microbes to embryos and semen stored in the vapour phase of liquid nitrogen in dry shippers. Cryobiology 2005;50:206-10

[13] Lim JJ, Shin TE, Song SH, Bak CW, Yoon TK, Lee DR. Effect of liquid nitrogen vapor storage on the motility, viability, morphology, deoxyribonucleic acid integrity, and mitochondrial potential of frozen-thawed human spermatozoa. Fertil Steril 2010;94: 2736-41.

[14] Punyatanasakchai P, Sophonsritsuk A, Weerakiet S, Wansumrit S, Chompurat D. Comparison of cryopreserved human sperm in vapor and liquid phases of liquid nitrogen: effect on motility parameters, morphology, and sperm function. Fertil Steril 2008;90:1978-82.

[15] Cobo A, Romero JL, Pérez S, de los Santos MJ, Meseguer M, Remohí J. Storage of human oocytes in the vapor phase of nitrogen. Fertil Steril 2010;94:1903-07.

[16] AbdelHafez F, Xu J, Goldberg J, Desai N. Vitrification in open and closed carriers at different cell stages: assessment of embryo survival, development, DNA integrity and stability during vapor phase storage for transport. BMC Biotechnol 2011;30;11-29.

[17] Pursel, VG, Johnson LA. Freezing of boar spermatozoa: fertilizing capacity with concentrated semen and a new thawing procedure. J Anim Sci 1975;40:99102.

[18] Martinez EA, Angel MA, Cuello C, Sanchez-Osorio J, Gomis J, Parrilla I, et al. Successful non-surgical deep uterine transfer of porcine morulae after 24 hour culture in a chemically defined medium. PLoS One. 2014; 9:e104696. 
1 [19] Funahashi H, Ekwall H, Rodriguez-Martinez H. Zona reaction in porcine oocytes fertilized in vivo and in vitro as seen with scanning electron microscopy. Biol Repro 2000;63:1437-42.

[20] Stringgfellow DA. Recomendations for the sanitary handling of in vivo derived embryos. In: Stringfellow DA, Seidel SM, editors. Manual of the International Embryo Transfer Society, Savoy, Illinois: IETS 1998:79-84.

[21] Cuello C, Sanchez-Osorio J, Almiñana C, Gil MA, Perals ML, Lucas X, et al. Effect of the cryoprotectant concentration on the in vitro embryo development and cell proliferation of OPS-vitrified porcine blastocysts. Cryobioloy 2008;56:189-94.

[22] Petters RM, Wells KD. Culture of pig embryos. J Reprod Fertil 1993;48:61-73.

[23] Brison DR, Schultz RM. Apoptosis during mouse blastocysts formation: evidence for a role for survival factors including transforming growth factor alpha. Biol Reprod 1998; 56,1088-96.

[24] Byrne AT, Southgate J, Brison DR, Leese HJ. Analysis of apoptosis in the preimplantation bovine embryo using TUNEL. J Reprod Fertil 1999;117:97-105.

[25] Sanchez-Osorio J, Cuello C, Gil MA, Parrilla I, Maside C, Almiñana C, et al. Vitrification and warming of in vivo-derived porcine embryos in a chemically defined medium. Theriogenology 2010;73:300-08.

[26] Gomis J, Cuello C, Sanchez-Osorio J, Gil MA, Parrilla I, Angel MA, et al. Forskolin improves the cryosurvival of in vivo-derived porcine embryos at very early stages using two vitrification methods. Cryobiology 2013;66:144-50.

[27] Cuello C, Sanchez-Osorio J, Almiñana C, Gil MA, Parrilla I, Roca J, et al. Superfine open pulled straws vitrification of porcine blastocysts does not require 
pretreatment with cytochalasin B and/or centrifugation. Reprod Fertil Dev 2010;22;808-17.

[28] Beebe LF, Cameron RD, Blackshaw AW, Keates HL. Changes to porcine blastocysts vitrification methods and improved litter size after transfer. Theriogenology 2005;64:879-90.

[29] Martinez EA, Martinez CA, Nohalez A, Sanchez-Osorio J, Vazquez JM, Roca $\mathrm{J}$, et al. Nonsurgical deep uterine transfer of vitrified, in vivo-derived, porcine embryos is as effective as the default surgical approach. Sci Rep 2015;5:10587.

[30] Cuello C, Martinez CA, Nohalez A, Parrilla I, Roca J, Gil MA, et al. Effective vitrification and warming of porcine embryos using a $\mathrm{pH}$-stable, chemically defined medium. Sci Rep 2016;6:33915.

[31] El-Gayar M, Gauly M, Holtz W. In vitro and in vivo survival of mouse blastocysts after repeated vitrification with the open pulled straw (OPS) method. Cryo Letters 2010;31:454-59.

[32] Polge, C. The freezing of mammalian embryos: perspectives and possibilities. Ciba Found Symp 1977;18-20;3-18.

[33] Nagashima H, Kashiwazaki N, Ashman RJ, Grupen CG, Nottle MB. Cryopreservation of porcine embryos. Nature, 1995:30:374-416.

[34] Hardy K, Handyside AH, Winston RM. The human blastocyst: cell number, death and allocation during late preimplantation development in vitro. Development 1989;107:597-604.

[35] Cuello C, Berthelot F, Delaleu B, Venturi E, Pastor LM, Vazquez JM et al. The effectiveness of the stereomicroscopic evaluation of embryo quality in vitrifiedwarmed porcine blastocysts: an ultrastructural and cell death study. Theriognelogy 2007;67: 970-82. 
1 [36] Hale AJ, Smith CA, Sutherland LC, Stoneman VE, Longthorne V, Culhane

2

3

4

5

6

7

8

9

10

11

12

13

14

15

16

17

18

19

20

21

22

23

24

25

AC,et al. Apoptosis: molecular regulation of cell death. Eur J Biochem 1996;236:1-26.

[37] Fabian D, Gjorret JO, Berthelot F, Martinat-Botté F, Maddox-Hyttel P. Ultrastructure and cell death of in vivo derived and vitrified porcine blastocysts. Mol Reprod Dev 2005;70:155-65.

(1)

8

(1)




\section{Figure legends}

2

3 Fig. 1: Schematic representation of the experimental design used in Experiment 1.

5 Fig. 2. Representative bright field images showing compacted-cavitating morulae and

6 unhatched blastocysts freshly collected (A and C, respectively) and at $24 \mathrm{~h}$ of in vitro

7 culture after vitrification-warming (B and D, respectively). Scale bar is $100 \mu \mathrm{m}$.

8

9 Fig. 3. Post-warming hatching rates at $48 \mathrm{~h}$ of culturing of re-vitrified (RVW) blastocysts derived from vitrified compacted or cavitating morulae (CCMs) and unhatched blastocysts (UBLs). Control embryos were fresh (not vitrified) CCMs and

12 UBLs cultured in vitro for $48 \mathrm{~h}$. The number of embryos evaluated is in parentheses. Different letters indicate significant differences $(\mathrm{P}<0.01)$.

Fig. 4: Schematic representation of the experimental design used in Experiment 2.

Fig. 5. Post-warming survival rates. Survival rates at $24 \mathrm{~h}$ post-warming of compacted or cavitating morulae (CCMs) and unhatched blastocysts (UBLs) that were vitrified and warmed and then stored for three days in a dry shipper or in a liquid nitrogen tank (control). Numbers of embryos used in parentheses.

Fig. 6. Post-warming embryonic development. A) Summary of the developmental stages at $24 \mathrm{~h}$ post-warming of compacted or cavitating morulae (CCMs) and unhatched blastocysts (UBLs) that were vitrified and warmed and then stored for three days in a dry shipper ( $\mathrm{N}=48$ and 46, respectively) or in a liquid nitrogen tank (control; $\mathrm{N}=56$ and 
142 , respectively). The developmental stage was scored according to the following

2 classes: 1, early blastocyst; 2, full blastocyst; 3 expanded blastocyst; 4, peri-hatching

3 blastocyst; 5, hatched blastocyst. Values are expressed as the mean \pm SD. B) Frequency

4 distribution of the different embryonic developmental stages achieved at $24 \mathrm{~h}$ post-

5 warming by compacted or cavitating morulae (CCMs) and unhatched blastocysts

6 (UBLs) that were vitrified and warmed and then stored for three days in a dry shipper

7 (DS) or in a liquid nitrogen tank (control).

8

9 Fig. 7. Post-warming total cell number and apoptotic index. A) Representative 10 fluorescence images of TUNEL staining of a vitrified blastocyst stored in a dry shipper 11 for three days. The Hoechst fluorophore stains all nuclei and displays blue fluorescence.

12 TUNEL stains nuclei with DNA fragmentation and displays green fluorescence. Bar: 50 $\mu \mathrm{m}$. B) Total cell number and apoptotic index at $24 \mathrm{~h}$ post-warming in the resulting blastocysts from vitrified and warmed compacted or cavitating morulae (CCMs) and unhatched blastocysts (UBLs) stored for three days in a dry shipper or in an LN tank (control). The number of embryos analyzed is in parentheses. Values are expressed as the mean \pm SD. Different letters indicate significant differences $(\mathrm{P}<0.05)$. 
1 Table 1. Survival rates of vitrified and warmed (VW) and subsequently re-vitrified and

2 warmed (RVW) embryos at $24 \mathrm{~h}$ post-warming.

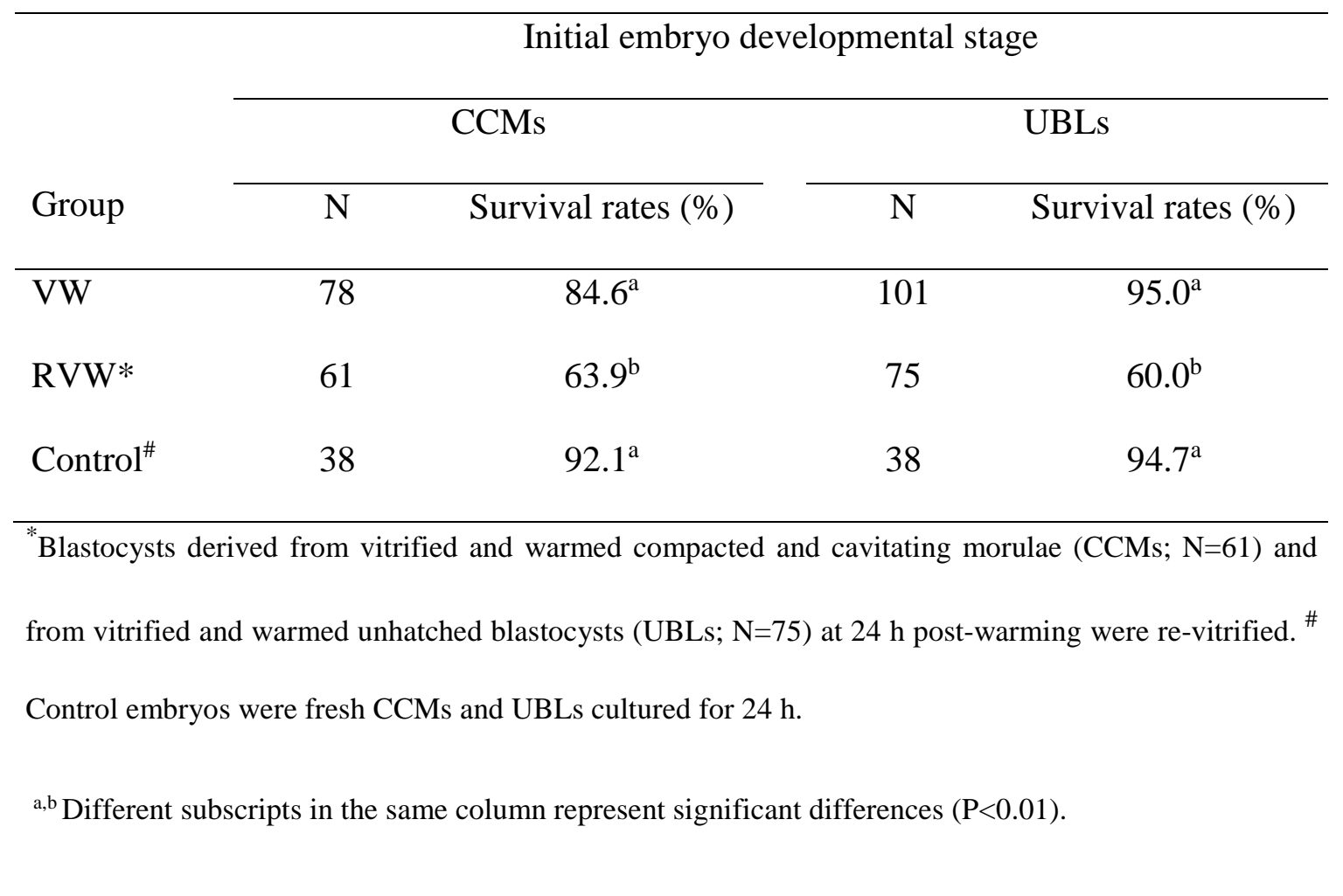




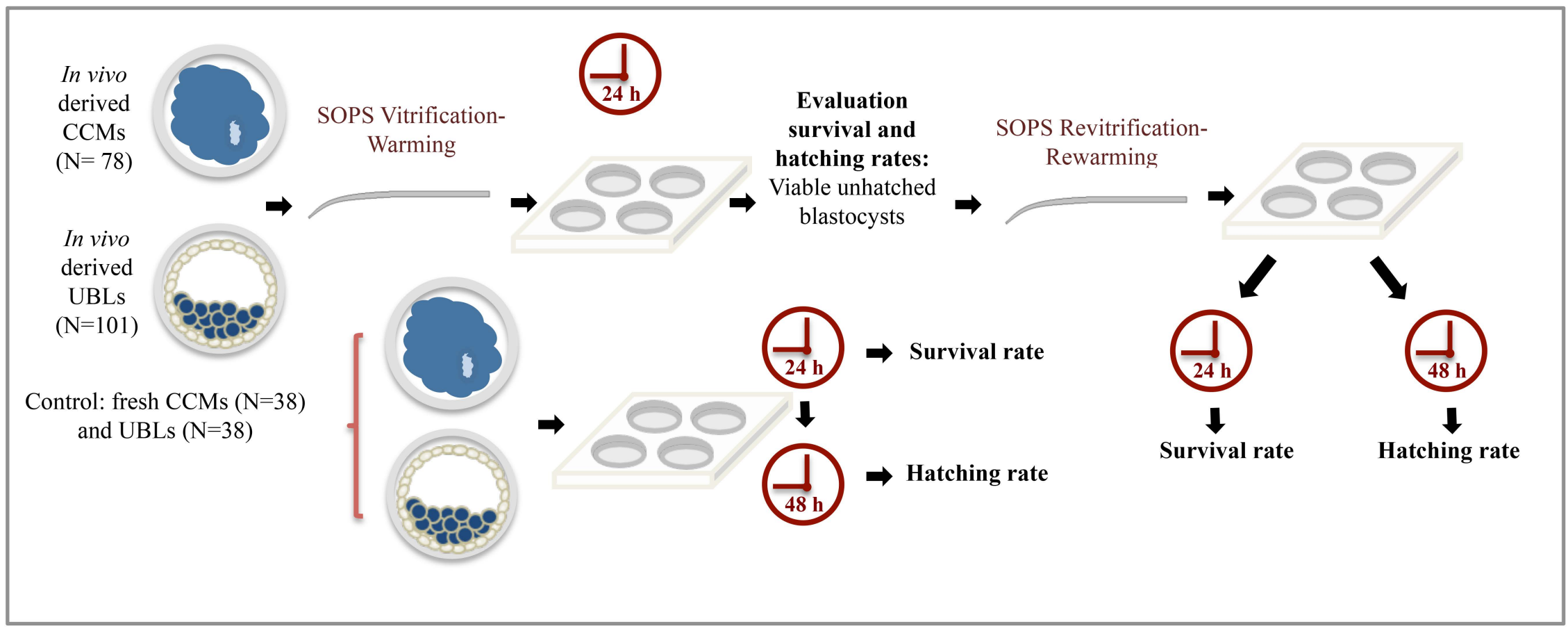


100

90.0 (b)

80

60

$\begin{array}{ll}50.3 & 50.8\end{array}$

40

0

(38) 


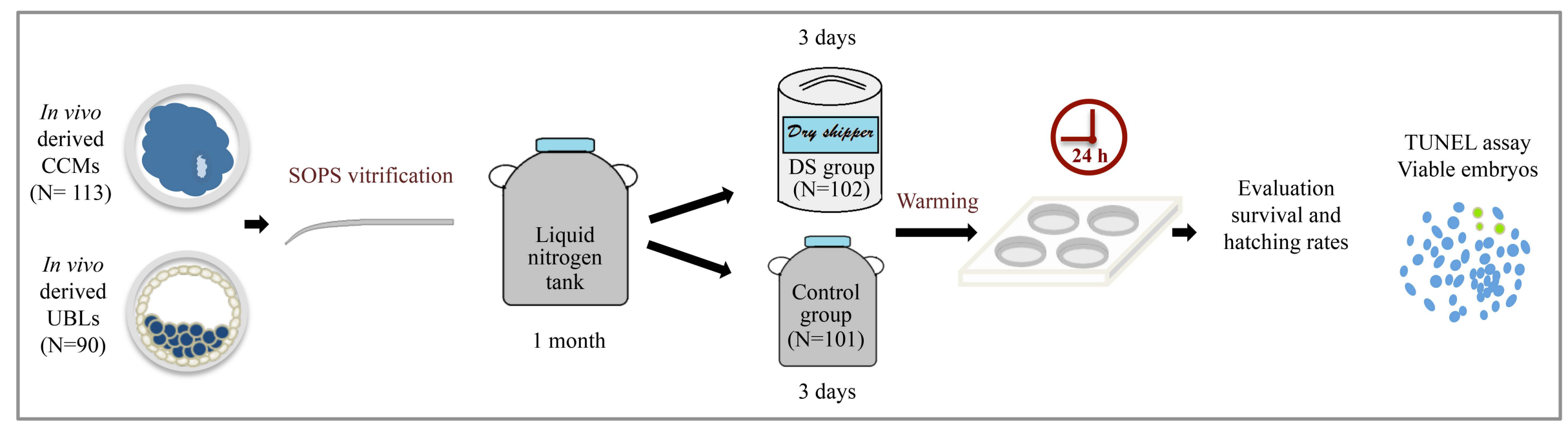


$(\%)$

100

80
60
40
20
0
- Dry shipper Control

95.8

94.9

88.8

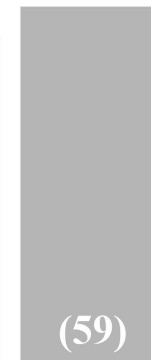

(54)
(48)

100

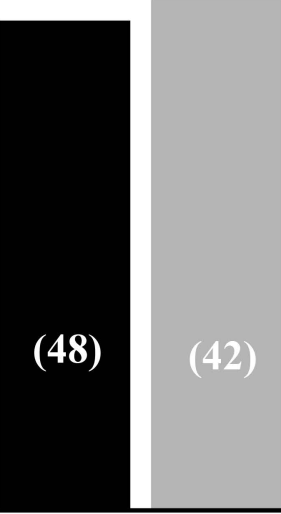

UBLs 
(B) Blastocyst stages

$\square$ Early $\square$ Full $\square$ Expanded $\square$ Perihatching $\square$ Hatched
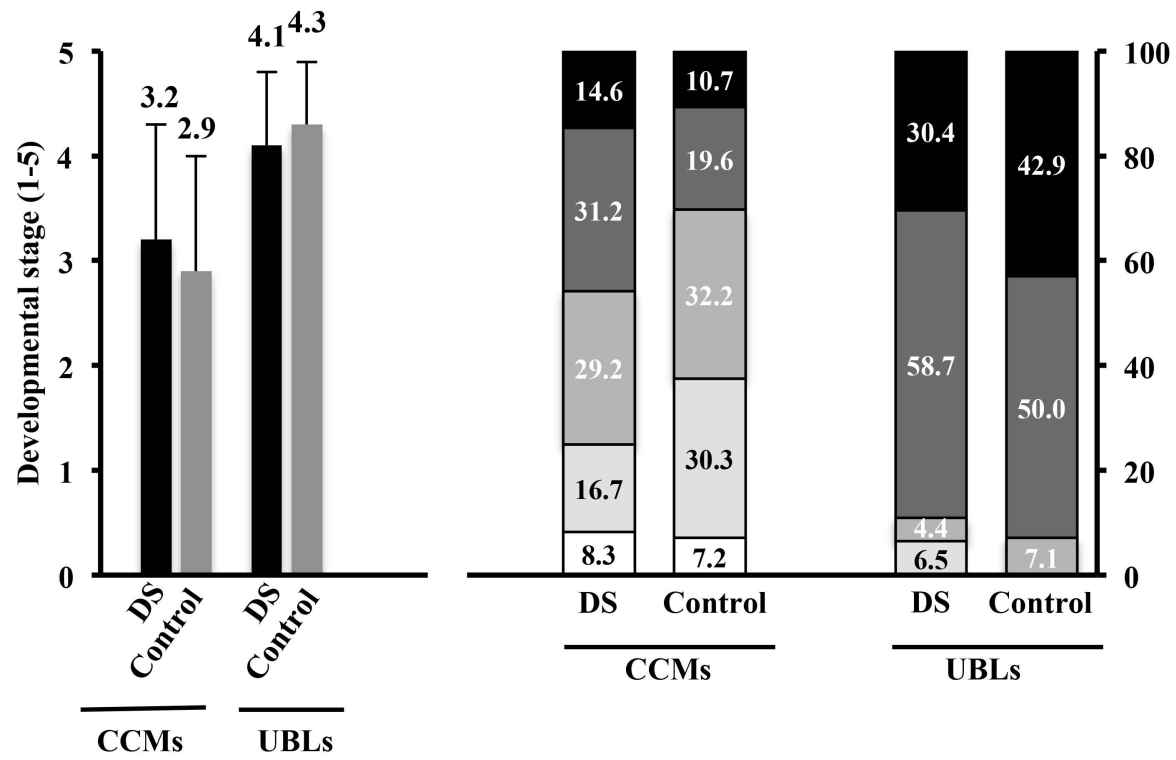

(\%) 
A

Hoechst 33342

TUNEL
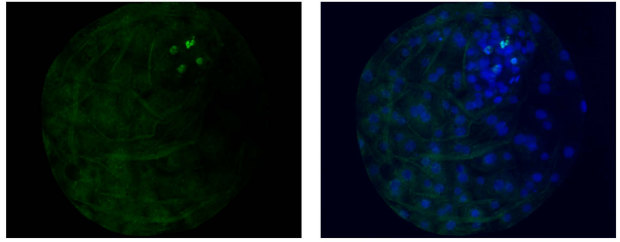

B

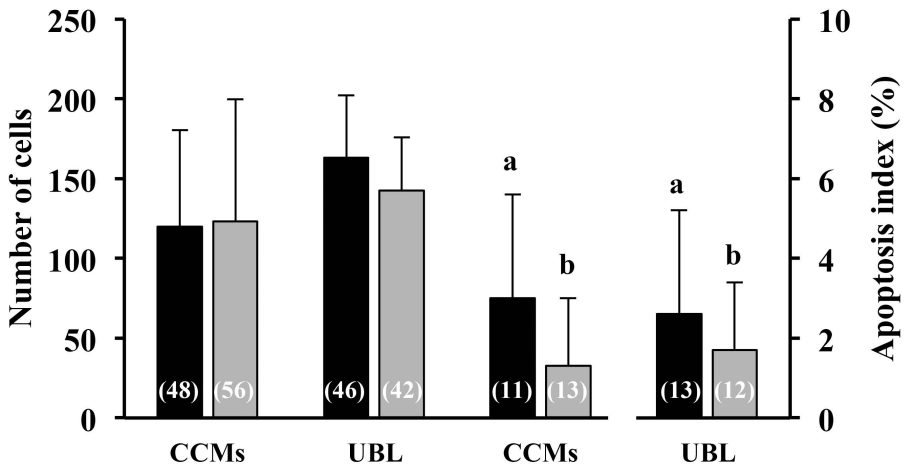

